\title{
Examining Workcell Kinematic Chains to Identify Sources of Positioning Degradation
}

\author{
Alexander Klinger ${ }^{1}$, Brian A. Weiss ${ }^{2}$ \\ ${ }^{1,2}$ National Institute of Standards and Technology (NIST), Gaithersburg, Maryland, 20899, USA \\ alexander.klinger@nist.gov \\ brian.weiss@nist.gov
}

\begin{abstract}
Automated industrial workcells are becoming increasingly complex and varied due to greater accessibility of advanced robotic and sensing technologies. Degradation monitoring and diagnostics must advance to reduce the impact of increased system complexity on troubleshooting faults and failures and to optimize system operations. A new methodology is being developed for the design and implementation of monitoring kinematic chains commonly found in robot workcells. This method will enable the identification of degraded components which contribute to relative positioning accuracy error between moving objects, tools, devices, and other components. The proposed methodology is being developed and tested on a six degree of freedom industrial robot arm workcell use case developed at the National Institute of Standards and Technology (NIST). Industrial robot users and integrators can use this method to examine the kinematic chains within their workcells and design a key position monitoring implementation. With the added key position monitoring, degradations can be identified at a designed resolution allowing for enhanced maintenance planning and production control. The methodology will be extended to other manufacturing workcells in the future.
\end{abstract}

\section{INTRODUCTION}

Robotics, along with supporting sensing and automation technologies, are actively used for a variety of tasks throughout the manufacturing industry (Ahmad \& Plapper, 2016; Chen, Fuhlbrigge, \& Li, 2008; Shi, Wang, \& Li, 2007). As robotic and sensor technologies have evolved, the diversity and complexity of use cases has grown. There is a need to better understand the health, and consequently, the wear of these automation systems, as the manufacturing community increases their reliance on advanced technologies. Systems can be maintained more efficiently,

\footnotetext{
Alexander Klinger et al. This is an open-access article distributed under the terms of the Creative Commons Attribution 3.0 United States License, which permits unrestricted use, distribution, and reproduction in any medium, provided the original author and source are credited.
}

leading to a more robust process, if/when advanced health information is generated.

A critical element of industrial robot workcells is reliable (inclusive of repeatability) positioning. In many robot workcells, a six degree of freedom (6DOF) industrial robot arm is used as a macro positioner for an end effector (i.e., tooling mounted to the tool flange of the robot). The tool may be a gripper in a material handling application or it could be a welding gun, paint applicator, or tooling that is specific to other operations (Agheli, Qu, \& Nestinger, 2014; Chen et al., 2008; Weiss \& Klinger, 2017). A workcell may be configured such that end effectors are changed during operations to enable a single robot to serve as the macro positioner for multiple tasks. Reliability of positioning accuracy is required when using industrial robots.

Researchers from the National Institute of Standards and Technology (NIST) are focused on developing the necessary measurement science to verify and validate monitoring, diagnostic, and prognostic technologies to promote enhanced maintenance and control strategies within manufacturing operations (Pellegrino, Justiniano, Raghunathan, \& Weiss, 2016; Weiss et al., 2015). Part of this effort is focused on measuring technologies supporting 6DOF robot workcells (Klinger \& Weiss, 2018; Weiss \& Klinger, 2017). This article presents NIST's development of a new methodology for the design and implementation of monitoring kinematic chains commonly found in robotic workcells. This method will enable the identification of degraded components that contribute to relative positioning accuracy errors between moving objects, tools, devices and other components. The proposed methodology is being developed and tested on a 6DOF industrial robot arm workcell use case developed at NIST. This methodology will offer a low-cost, minimally invasive means for a manufacturer to quickly assess the health of their robot workcell and ascertain where in the kinematic chain positional errors are originating. Ultimately, this research will be used to promote industry-driven standards to verify and validate health monitoring of workcell technologies (Vogl, Weiss, \& Donmez, 2014a, 2014b; Brian A Weiss, Alonzo, \& Weinman, 2017). 
The remainder of this paper is organized as follows. Section 2 presents background on the need for identifying points/areas of degradation within the kinematic chain along with some prior work. Section 3 provides a detailed discussion of the proposed kinematic chain analysis methodology. Section 4 discusses the kinematic chain inspection process to identify degradations. Section 5 highlights future work. Finally, Section 6 concludes the paper and acknowledges future work.

\section{BACKGROUND}

The Prognostics and Health Management (PHM) community is focused on advancing monitoring, diagnostic, and prognostic capabilities to maintain and/or improve asset availability, product/process quality, and productivity of a range of operations, including those within manufacturing. PHM has been actively applied to manufacturing factories with varying success (Helu \& Weiss, 2016; Jin, Siegel, et al., 2016; Jin, Weiss, Siegel, \& Lee, 2016). This has included the development and implementation of numerous strategies to minimize reactive maintenance and optimize the balance between preventive and predictive maintenance (Lee, Lapira, Bagheri, \& Kao, 2013; Lee, Ni, Djurdjanovic, Qiu, \& Liao, 2006; Peng, Dong, \& Zuo, 2010; Vogl, Weiss, \& Helu, 2016).

Most manufacturing robot workcells can be considered complex systems of systems. The overall physical system can be broken down into sub-systems, components, subcomponents, etc. Decomposing the physical elements of a robot workcell into a hierarchy of elements offers a means of defining boundaries that can drive maintenance activities. This physical hierarchy can be connected with functional and informational hierarchies to provide a means of understanding complex relationships and identifying metrics and measures of workcell health (Sharp \& Weiss, 2018; Weiss \& Qiao, 2017; Weiss, Sharp, \& Klinger, 2018). The more complex a system, the more critical it becomes to understand its inherent relationships to see how the state of mechanical degradation of physical elements impacts process performance.

Research has been performed in monitoring the health, including positioning, of a robot, itself, separate from the workcell. Vision and laser tracker systems are two types of technologies that have been paired with PHM algorithms to enhance health awareness (Qiao \& Weiss, 2017; Spiewak, Zaiss, \& Ludwick, 2013; Wan, Song, Xu, Liu, \& Chen, 2018). There is a need to expand the health monitoring capabilities beyond the robot, itself, and to consider the entire robot workcell. Understanding and monitoring the positioning health of all elements of kinematic chains is a necessary part of a comprehensive PHM system for robot workcells. Prior research has been performed in monitoring the health of robot workcells, yet research has not been uncovered that focuses on specifically monitoring kinematic chain health. Existing robot monitoring approaches present unique solutions that are either too high-level and lacking specific guidance (Lopez-Mellado \& Alami, 1990) or rely upon potentially costly technology (Jokinen \& Lastra, 2016; Novak-Marcincin, Torok, Janak, \& NovakovaMarcincinova, 2014). NIST personnel believe that monitoring and analyzing the kinematic chain for positional degradation can be done in a cost-effective and minimallyintrusive manner to increasing benefit for the robot workcell owners and users.

\section{Kinematic Chain Analysis}

Kinematic chains can be documented at various levels of complexity for the same mechanism. At the most detailed level, a kinematic chain contains all bodies, including components and sub-components, fasteners and other parts. The documentation of some kinematic chains may only include links between actuated joints or express entire actuated assemblies as single links.

It is beneficial to observe multiple points along a kinematic chain in a serial manipulator to monitor positioning accuracy and identify the source of positioning errors. By inspecting repeatability at multiple points along a chain, the source of repeatability degradation can be narrowed down to the component(s) located between adjacent inspection points. To facilitate this, a way of representing a kinematic chain is needed that provides sufficient detail to choose relevant inspection points to meet monitoring objectives. To develop this representation, a suggested starting point is to document the chain at the component level while identifying the relationships between components through their interfaces.

The kinematic chain is made of links which can be connected to other links or a reference frame. Each component or element of the system is considered a link in the kinematic chain. Each link has a left-hand side (LHS) and a right-hand side (RHS) which correspond to that component's interfaces with other links in the chain. It is important to note that LHS and RHS elements are physical pieces of the link, itself. For example, if a robot is a link, the LHS would likely be the robot's base since that would be physically connected to a mounting surface and the RHS would likely be the robot's tool flange since this is where an end effector would be joined. At the LHS and RHS boundaries of each link, permanent connections are represented by brackets '[,]' and intermittent connections with parenthesis '(,)'. For example, a gripper, which is permanently fixed to a robot tool flange, will have a '[' on its LHS corresponding to the permanent connection to a robot and a ')' on its RHS corresponding to an intermittent connection to a part as shown in Figure 1.

When individual links are joined to form a kinematic chain, the physical interfaces, represented by the LHS and RHS of adjacent links and the type of connection (permanent or intermittent), are easily identifiable as seen in Figure 2. A reference frame must exist at a boundary at either the far left 
(a) Link Representation

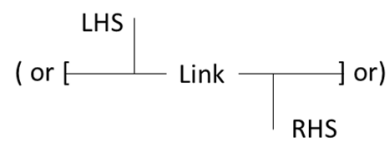

(b) Example: Robot

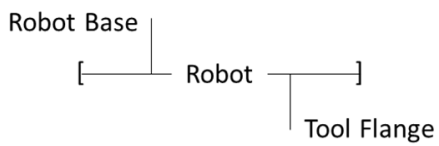

(c) Example: Gripper

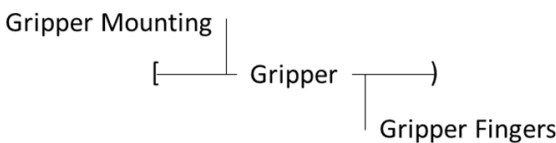

Figure 1. Kinematic chain individual link representation: (a) generic link, (b) example of a robot with permanent connections at both interfaces, and (c) example of a gripper with intermittent connection on RHS

or far right (or both) of the chain to provide a basis of measurement. Using this convention also allows for the kinematic chain to be represented in text. A single link can be represented as [LHS, Link, RHS] or more simply [Link]. For example, the chain in Figure 2(a) can be represented as 'Reference] [LHS 1, Link 1, RHS 1] [LHS 2, Link 2, RHS 2)' or in a simpler form 'Reference] [Link 1] [Link 2).'

A variety of components are typically chosen by a machine integrator for specific tasks during industrial robot workcell design and integration. This variety can pose a challenge in expressing the range of possible configurations. At

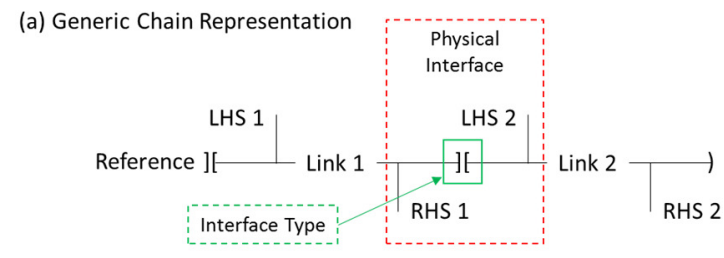

(b) Example: Robot and Gripper

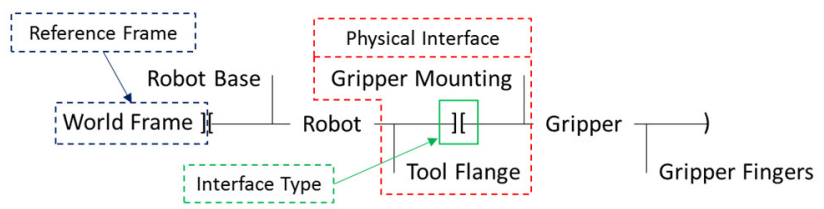

Figure 2. Kinematic chain representation of two links with key features identified. (a) Generic representation, (b) robot and gripper example. minimum, every robotic workcell includes an industrial robot arm and an end effector. These components will serve as the starting point of the kinematic chain as shown in Figure 2(b). Not all kinematic chains remain intact throughout the entirety of a manufacturing process. In use cases where there are multiple, swappable end effectors, multiple chains will exist throughout the overall process. Likewise, an end effector may perform two or more functions. Each function will generate a unique kinematic chain which includes the interactions that are specific to those functions. Additional chains will need to be documented and analyzed in these scenarios.

Beyond the robot arm and end effector, additional workcell components are considered part of the kinematic chain if they impact the positional accuracy of any component or action within the process. These components include elements that are not actuated or actively controlled yet are critical in the relative positioning of parts and equipment during operation. For example, a hard stop may be used to orient a part. If the hard stop is not properly positioned, the part will not be oriented properly when aligned with the stop. Therefore, the hard stop should be included in the kinematic chain. Additional components can also include parts being worked on and the fixturing that is holding them.

\subsection{NIST Use Case Kinematic Chains}

A use case and testbed with a material handling robot and a path-following robot were developed and built at NIST to serve as a platform to support robotic workcell PHM research (Klinger \& Weiss, 2018; Brian A Weiss \& Klinger, 2017). The use case begins with the material handling robot, with a gripper end effector, picking parts from an input, and placing them on a work fixture (Figure 3(a)). The path following robot, with a pen holding end effector, then draws on the part (Figure 3(b)). Once the drawing operation is complete, the part is then picked by the material handling robot and placed in an output location (Figure 3(c)).

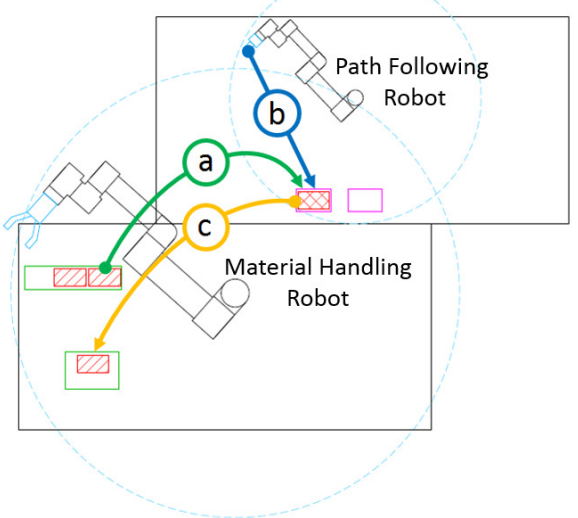

Figure 3. NIST use case: (a) Material handling robot moves a part a work fixture. (b) Drawing robot draws on the part. (c) Material handling robot moves the completed part to the output 
(a) Use Case Kinematic Chain - Graphic Representation

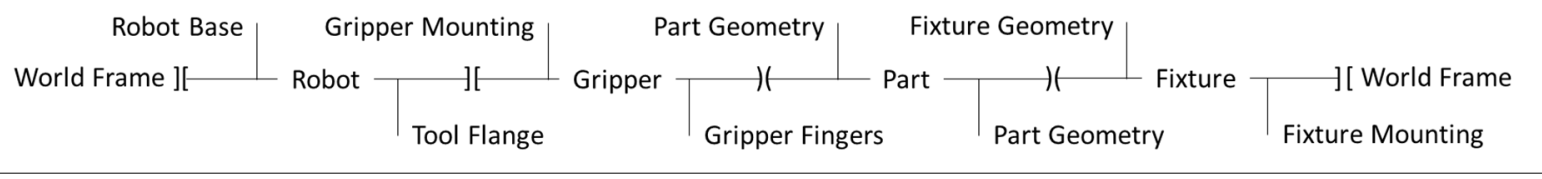

(b) Use Case Kinematic Chain - Long Text Representation

World Frame ][ Robot Base, Robot, Tool Flange ][ Gripper Mounting, Gripper, Gripper Fingers )... ...( Part Geometry, Part, Part Geometry )( Fixture Geometry, Fixture, Fixture Mounting ][ World Frame

(c) Use Case Kinematic Chain - Short Text Representation

World Frame ][ Robot ][ Gripper )( Part )( Fixture ][ World Frame

Figure 4. The NIST use case material handling robot kinematic chain: (a) graphical representation, (b) long-form text representation, (c) short-form text representation.

As seen in Figure 4, for this use case, the world frame is the reference frame and is set by the table which the robot and fixtures are rigidly mounted on. The robot is the first link, followed by the gripper, then the part, and finally the fixture. The critical relationships in this kinematic configuration include the robot base to a table - a permanent connection shown in Figure 5(a), the robot tool flange to the gripper mounting - a permanent connection shown in Figure 5(b), the gripper fingers to the part - an intermittent connection shown in Figure 5(c), the part to the fixture - another intermittent connection, and the fixture to the table - a permanent connection highlighted in Figure 5(d).

When the robot is in position to pick or place a part there is a constrained relationship between the part and the gripper, as well as the part and the fixture. The robot must position the gripper in a designed relationship to the fixture (and part) during part pick and placement because of these physical constraints. The interfaces of the intermittent connection between the gripper and part are shown as the RHS of the gripper (gripper fingers) and the LHS of the part (part geometry) respectively. Likewise, interfaces of the intermittent connection between the part and the fixture are shown as the geometries of the part and the fixture.

\section{INSPECTING THE KINEMATIC CHAIN TO IDENTIFY DEGRADATION}

Identifying and tracking degradation of workcell components is possible by measuring positioning repeatability of key points along kinematic chains. By inspecting the positioning repeatability of individual components relative to a reference frame, components which have degraded in their ability to position repeatedly beyond a design limit can be identified. The proposed inspection method can treat kinematic chains as open loop chains due to the nature of serial manipulators. There may be workcell configurations where the kinematic chain is represented as a closed loop, as in the NIST use case example, but by inspecting individual points along the chain

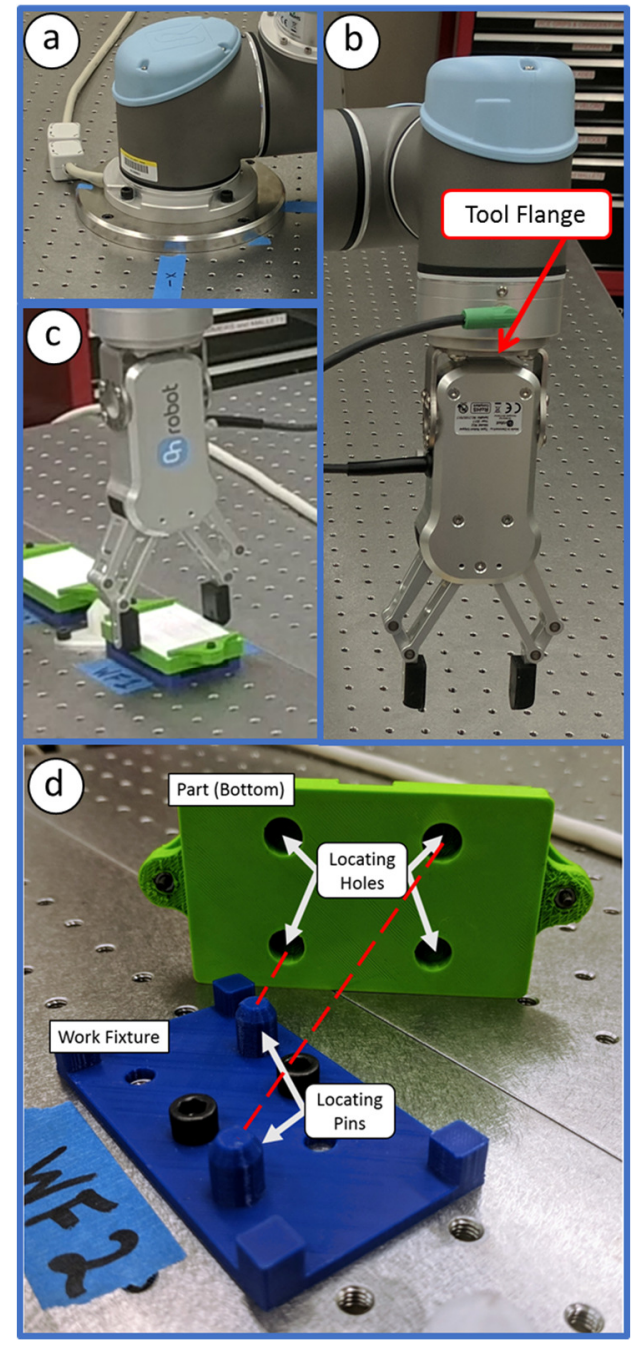

Figure 5. NIST use case material handling robot relationships: (a) robot base to the table, (b) robot tool flange to gripper mounting, (c) gripper fingers to part when on fixture, (d) fixture to table. 
this does not pose an issue. In cases where the chain is a closed loop, which is typically due to multiple components interfacing directly with the same reference frame as the robot, inspections may need to be carried out working from both the LHS and RHS reference frames. This will be discussed further and through example with the NIST use case.

Inspection of components starts at a reference frame. The measurement sensor(s) is in a known location to the reference frame and is assumed to be positioned and operating properly. Working left-to-right in the kinematic chain, the sensor(s) is fixed in the left most reference frame. Inspection begins at the RHS of the first component (link) with the testing of the position of the RHS of the component at a taught position. This assesses if the first component is maintaining sufficient repeatability to succeed. If the test is successful, then all parts of the first link are being positioned nominally. This is represented in Figure 6(a). After the first component is tested to be within specification the process continues, from left to right, by inspecting each component to confirm that each link is achieving its nominal test positions. After the RHS of the second link is tested successfully, the interface between the first and second link can be declared nominal and all parts of the second link are positioned nominally. This is represented in Figure 6(b).

(a) Link 1 Inspection

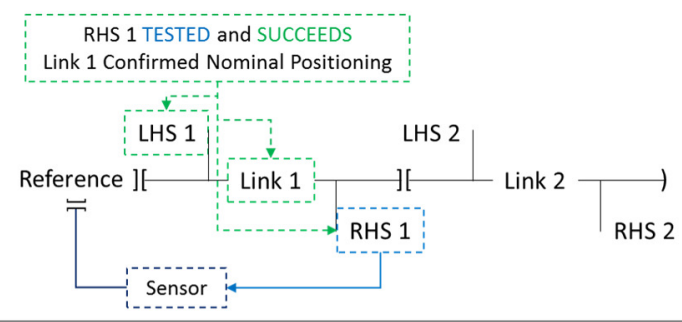

(b) Link 2 Inspection RHS 2 TESTED and SUCCEEDS Link 1 to Link 2 Interface Confirmed Nominal Link 2 Confirmed Nominal

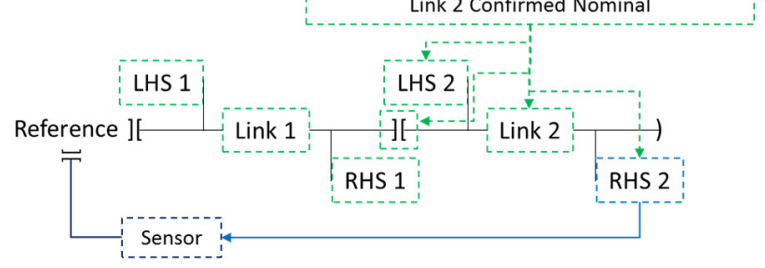

Figure 6. Annotated generic link inspection method.

The decision of whether a component is being positioned nominally and a test is successful will depend on the designed allowance in the system. Due to tolerance stacking, it is not unusual to expect the allowance of components further from the reference in the kinematic chain to be higher and must be considered when choosing the sensor(s) used for inspection. It can also be beneficial to choose inspection points other than the RHS of links in the kinematic chain depending on the workcell. To help choose these points, an analysis of modes of positioning degradation is recommended.

\subsection{Identifying Positioning Degradation Modes Along the Kinematic Chain}

Likely sources of positional relationship issues can be identified once the kinematic chain and the interactions between components are mapped. The type and cause of positioning error will vary based upon the components within the workcell. Degradation modes can be found by assessing each link and the interactions between the links.

A link can be actuated, like a robot, or rigid, like a part. These two types of links can degrade in many ways which can be classified as either rigid body deformations, or degraded actuation and positioning. With an actuated link, such as a robot arm, if any joint in the robot is not positioning itself correctly, the tool flange and end effector will not be positioned correctly. These positioning errors can present themselves instantaneously after crashes or over time after wear. Likewise, deformation of rigid bodies can occur instantaneously or over time. For example, gripper fingers may bend when colliding with a foreign object in the workcell as compared to the fingers fatiguing and bending over time after repeated use. Each link is susceptible to degradation and will influence how that component and the rest of the workcell performs.

In addition to the positioning error modes from the links, themselves, the interactions between the links must be studied to assess the impact of link degradation on the relationships. Many different interactions can exist within the workcell. Couplings between components are either permanent or intermittent. Generally, the interface between two components is critical when the components' interaction is intermittent. This type of interaction requires the components to be in their designed positions and may involve a specific actuation for coupling. This means that if a component is positioned improperly, the geometry is inaccurate or unexpected, or a component cannot actuate as intended to successfully execute the interaction, the process can be considered degraded and may not perform nominally. For example, when a part is being grasped by a gripper, the part must have the expected geometry and be properly positioned for the gripper which must be in the expected position and actuate as designed to successfully grasp the part.

\subsection{Identifying Positioning Degradation Modes in the NIST Use Case}

A variety of positioning degradation modes are identified within the NIST use case. Degradation modes are identified for the components of the kinematic chain as well as the interactions between components. Starting at the world frame and examining the kinematic chain of Figure 4, moving left to right, modes are identified. Table 1 summarizes the 
identified degradation modes. The modes internal to components, including robot wear, gripper wear, bad parts, and fixture wear are expressed as between the LHS and RHS of their respective components. The connection is assumed as the only failure mode between two links that are permanently fixed to one another and other modes are considered as part of the components. For the intermittent interactions between the part and the gripper, and the part and the fixture, any of the components can contribute to positioning error leading to multiple modes listed.

Table 1. Use case relationships table.

\begin{tabular}{|c|c|}
\hline Kinematic Chain Section & $\begin{array}{l}\text { Degradation } \\
\text { Mode(s) }\end{array}$ \\
\hline World Frame ][ Robot Base & Loose Connection \\
\hline $\begin{array}{l}\text { [Robot Base, ROBOT, Robot Tool } \\
\text { Flange] }\end{array}$ & Robot Wear \\
\hline Robot Tool Flange ][ Gripper Mounting & Loose Connection \\
\hline $\begin{array}{l}\text { [Gripper Mounting, GRIPPER, Gripper } \\
\text { Fingers] }\end{array}$ & Gripper Wear \\
\hline Gripper Fingers )( Part Geometry & $\begin{array}{l}\text { Gripper Wear, } \\
\text { Bad Part* }\end{array}$ \\
\hline [ PART ] & Bad Part* \\
\hline Part Geometry )( Fixture Geometry & $\begin{array}{l}\text { Bad Part*, } \\
\text { Bad Fixture** }\end{array}$ \\
\hline $\begin{array}{l}\text { [Fixture Geometry, Fixture, Fixture } \\
\text { Mounting] }\end{array}$ & Bad Fixture** \\
\hline Fixture Mounting ][ World Frame & Loose Connection \\
\hline
\end{tabular}

\subsection{Selection of Key Measurement Points to Identify Points / Areas of Degradation and Inspection Method}

Discrete points along the kinematic chain can be selected for inspection to detect positioning deviations from nominal or verify nominal positioning. These key measurement points need to be tested in one or more degrees of freedom to determine if they can be positioned within designed limits. Each point should be chosen to provide information on the source of a positioning error when measured in a specific order. As discussed in Section 3, a good starting point in a LHS-referenced kinematic chain is the RHS of each link in the chain. Inspecting the RHS of each link will provide insight into the repeatability of each component. However, if a component such as an end effector is actuated, it may be beneficial to inspect both the RHS and another point earlier in the kinematic chain. Inspecting before an actuation point of a particular component, the source of repeatability degradation can be isolated to within the component or to the connection between the component and the preceding component. Additionally, an actuated component can be inspected in multiple configurations or states to provide further isolation of degradation.

\subsection{Selection of Key Measurement Points in the NIST Use Case}

Applying this methodology to the NIST use case, the key points to be selected should be the robot tool flange, gripper fingers, and part geometry because they are the RHS of the components of the chain. Because the fixture link is directly connected to the world frame on its RHS, the LHS of the fixture link, fixture geometry, should be selected as a key point. Examining the gripper further, it is actuated and can have multiple states (i.e., open, gripping a part, closed) throughout its operations and can be inspected in more places than only the RHS. By selecting the gripper body as a key point before the finger actuation in the chain, the gripper mounting can be tested independently of the finger actuation. Likewise, testing the fingers in both an open and closed state, the operation and positing of the gripper fingers can be tested independently of the gripper mounting. To test the part geometry, the system must grasp an ideal part so the part position can be tested in-situ. To test the fixture geometry, a sensor can be mounted to or embedded in the fixture. These points are represented in Figure 7.

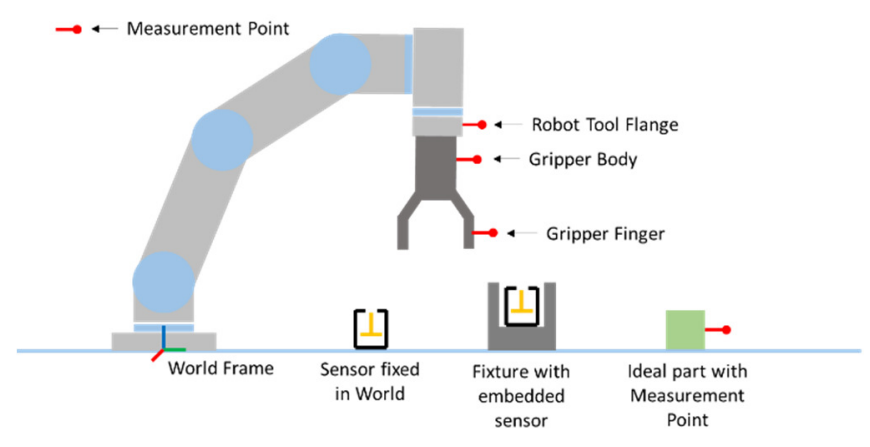

Figure 7. Use case key measurement point selection graphic.

Appropriate tolerancing or analysis thresholding can then be chosen for the pass / fail classification of each of the key points selected. Given the tolerance and monitoring requirements of the use case, appropriate sensors can be chosen. The key points can be tested in a sequence following the kinematic chain, left-to-right, once the sensors are integrated. The feedback from testing allows a series of binary decisions to be made to identify degraded components. Decision trees and testing criteria will be dependent on the use case. The decision tree and testing flow for the NIST use case can be seen in Figure 8. This series of tests can be performed at any interval and records kept to aide in the tracking of degradations and predicting future degradation. If the tests are done in the prescribed order, according to Figure 8 , a failed test will automatically indicate where, in the kinematic chain, a degradation is originating, providing a target component to repair or replace. After a failure of a test point, all points tested further along the kinematic chain will be unreliable without a full understanding of the cause of the failed test. 


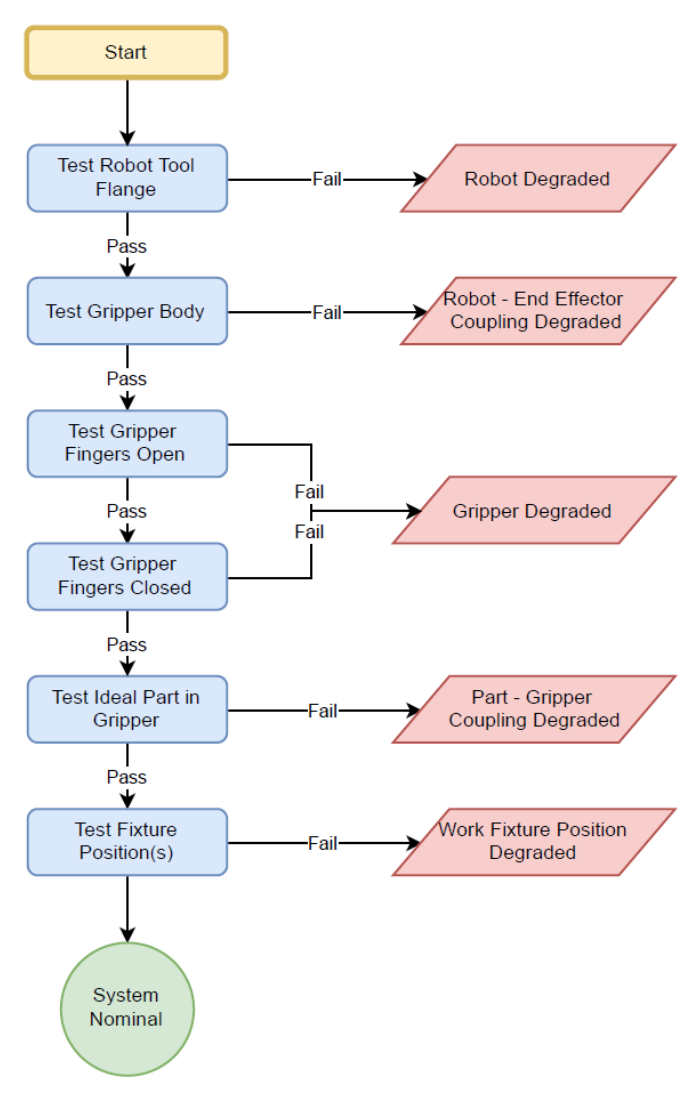

Figure 8. Binary decision tree to identify degraded component.

\section{FUTURE WORK}

Measuring or testing the selected key points along the kinematic chain can be done in many ways. Regardless of the specific details, there will be a sensor and a target, which the sensor measures. Measurements can be made through physical contact, visual imaging, or another type of measurement technology. The key characteristic to the sensor used for the kinematic inspection method outlined is that the sensor outputs information on the position of a specific point or object. Future work includes the assessment of measurement methods and sensors for examining robot workcell kinematic chains. This work will involve understanding the implications of using discrete and analog sensing methods, data collection strategies, and prognostic implications. This work will be done in parallel with the implementation of the discussed method on the NIST use case with commercially available and/or purpose-built sensors.

\section{Conclusion}

This paper presents a method to document kinematic chains within a robot workcell enabling the selection of key measurement points for testing to gain intelligence on workcell health. Testing of key measurement points along the kinematic chain can provide intelligence on the degradation of positioning of components. By choosing the measurement points with the help of a degradation mode analysis, a test or series of tests can be designed to identify the degraded component(s) which are potentially degrading the workcell's process. Initial efforts in this research are very promising where next steps are currently in process. This includes the development of a novel sensor to pair with the kinematic chain methodology to meet the effort's goals of providing a low-cost, minimally invasive means to identify degradations within the kinematic chain. Preliminary data sets will be captured upon integration of the novel sensor with the kinematic chain testing methodology. These initial data sets will offer critical insight as to the practical feasibility of kinematic chain methodology and the deployment/usage of the novel sensor.

\section{NIST DISCLAIMER}

The views and opinions expressed herein do not necessarily state or reflect those of NIST. Certain commercial entities, equipment, or materials may be identified in this document to illustrate a point or concept. Such identification is not intended to imply recommendation or endorsement by NIST, nor is it intended to imply that the entities, materials, or equipment are necessarily the best available for the purpose.

\section{REFERENCES}

Agheli, M., Qu, L., \& Nestinger, S. S. (2014). SHeRo: Scalable hexapod robot for maintenance, repair, and operations. Robotics and Computer-Integrated Manufacturing, $\quad 30(5), \quad$ 478-488. doi:10.1016/j.rcim.2014.03.008

Ahmad, R., \& Plapper, P. (2016). Safe and Automated Assembly Process using Vision Assisted Robot Manipulator. Procedia CIRP, 41, 771-776. doi:http://dx.doi.org/10.1016/j.procir.2015.12.129

Chen, H., Fuhlbrigge, T., \& Li, X. (2008). Automated industrial robot path planning for spray painting process: a review. Paper presented at the IEEE International Conference on Automation Science and Engineering, 2008. CASE 2008..

Helu, M., \& Weiss, B. A. (2016). The current state of sensing, health management, and control for small-tomedium-szed manufacturers. Paper presented at the ASME 2016 Manufacturing Science and Engineering Conference, MSEC2016.

Jin, X., Siegel, D., Weiss, B. A., Gamel, E., Wang, W., Lee, J., \& Ni, J. (2016). The present status and future 
growth of maintenance in US manufacturing: results from a pilot survey. Manufacturing Rev., 3, 10.

Jin, X., Weiss, B. A., Siegel, D., \& Lee, J. (2016). Present Status and Future Growth of Advanced Maintenance Technology and Strategy in US Manufacturing. International Journal of Prognostics and Health Management, 7 (Special Issue on Smart Manufacturing PHM)(Sp5), 18.

Jokinen, J., \& Lastra, J. L. M. (2016). Implementation of nonintrusive monitoring and fault diagnosis in industrial robot system. Paper presented at the 2016 IEEE 14th International Conference on Industrial Informatics (INDIN).

Klinger, A. S., \& Weiss, B. A. (2018). Robotic Work Cell Test Bed to Support Measurement Science for PHM. Paper presented at the 2018 ASME Manufacturing Science and Engineering Conference (MSEC), College Station, Texas.

Lee, J., Lapira, E., Bagheri, B., \& Kao, H.-a. (2013). Recent advances and trends in predictive manufacturing systems in big data environment. Manufacturing Letters, 1(1), 38-41.

Lee, J., Ni, J., Djurdjanovic, D., Qiu, H., \& Liao, H. (2006). Intelligent prognostics tools and e-maintenance. Computers in Industry, 57(6), 476-489.

Lopez-Mellado, E., \& Alami, R. (1990). A failure recovery scheme for assembly workcells. Paper presented at the Robotics and Automation, 1990. Proceedings., 1990 IEEE International Conference on.

Novak-Marcincin, J., Torok, J., Janak, M., \& NovakovaMarcincinova, L. (2014). Interactive Monitoring of Production Process with Use of Augmented Reality Technology. Applied Mechanics and Materials, $616,19$.

Pellegrino, J., Justiniano, M., Raghunathan, A., \& Weiss, B. A. (2016). Measurement Science Roadmap for Prognostics and Health Management for Smart Manufacturing Systems. NIST Advanced Manufacturing Seriess (AMS).

Peng, Y., Dong, M., \& Zuo, M. J. (2010). Current status of machine prognostics in condition-based maintenance: a review. The International Journal of Advanced Manufacturing Technology, 50(1-4), 297313.

Qiao, G., \& Weiss, B. A. (2017). Accuracy Degradation Analysis for Industrial Robot Systems. Paper presented at the ASME International Manufacturing Science and Engineering Conference, Los Angeles, California.

Sharp, M., \& Weiss, B. A. (2018). Hierarchical modeling of a manufacturing work cell to promote contextualized PHM information across multiple levels. Manufacturing Letters, 15, 46-49.

Shi, Y. H., Wang, G. R., Li, \& G. J. (2007). Adaptive robotic welding system using laser vision sensing for underwater engineering. In 2007 IEEE International
Conference on Control and Automation, Vols 1-7 (pp. 1795-1800).

Spiewak, S., Zaiss, C., \& Ludwick, S. J. (2013, November 15-21, 2013). High Accuracy, Low-Invasive Displacement Sensor (HALIDS). Paper presented at the ASME 2013 International Mechanical Engineering Congress and Exposition, IMECE 2013, San Diego, CA, United states.

Vogl, G. W., Weiss, B. A., \& Donmez, M. A. (2014a). Standards for prognostics and health management (PHM) techniques within manufacturing operations. Paper presented at the Annual Conference of the Prognostics and Health Management Society 2014, Fort Worth, Texas, USA.

Vogl, G. W., Weiss, B. A., \& Donmez, M. A. (2014b). Standards Related to Prognostics and Health Management (PHM) for Manufacturing (NISTIR 8012). Retrieved from Gaithersburg, Maryland, USA: http://dx.doi.org/10.6028/NIST.IR.8012

Vogl, G. W., Weiss, B. A., \& Helu, M. (2016). A review of diagnostic and prognostic capabilities and best practices for manufacturing. Journal of Intelligent Manufacturing. doi:10.1007/s10845-016-1228-8

Wan, A., Song, L., Xu, J., Liu, S., \& Chen, K. (2018). Calibration and compensation of machine tool volumetric error using a laser tracker. International Journal of Machine Tools and Manufacture, 124, 126-133.

Weiss, B. A., Alonzo, D., \& Weinman, S. D. (2017). Summary Report on a Workshop on Advanced Monitoring, Diagnostics, and Prognostics for Manufacturing Operations.

Weiss, B. A., \& Klinger, A. S. (2017). Identification of Industrial Robot Arm Work Cell Use Cases and a Test Bed to Promote Monitoring, Diagnostic, and Prognostic Technologies. Paper presented at the 2017 Annual Conference of the Prognostics and Health Management (PHM) Society, St. Petersburg, FL.

Weiss, B. A., \& Qiao, G. (2017). Hierarchical Decomposition of a Manufacturing Work Cell to Promote Monitoring, Diagnostics, and Prognostics. Paper presented at the ASME 2017 International Manufacturing Science and Engineering Conference (MSEC2017), Los Angeles, California.

Weiss, B. A., Sharp, M., \& Klinger, A. (2018). Developing a hierarchical decomposition methodology to increase manufacturing process and equipment health awareness. Journal of Manufacturing Systems.

Weiss, B. A., Vogl, G. W., Helu, M., Qiao, G., Pellegrino, J., Justiniano, M., \& Raghunathan, A. (2015). Measurement Science for Prognostics and Health Management for Smart Manufacturing Systems: Key Findings from a Roadmapping Workshop. Paper presented at the Annual Conference of the 
Prognostics and Health Management Society 2015, Coronado, CA.

\section{BIOGRAPHIES}

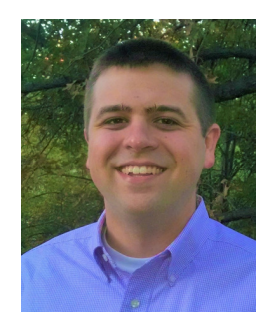

Alexander Klinger has a B.S. in Mechanical Engineering (2013) from Lehigh University, Bethlehem, Pennsylvania and a Masters of Engineering in Mechanical Engineering (2017) from the University of Maryland, College Park, Maryland. He is currently a mechanical engineer working on the Prognostics, Health Management and Control project within the Engineering Laboratory (EL) at the National Institute of Standards and Technology (NIST). Prior to his role at NIST, he was a mechanical engineer at the United States Postal Service (USPS) Engineering Headquarters, working on automation and robotic systems. He has also worked in various engineering roles in the manufacturing and entertainment industry.

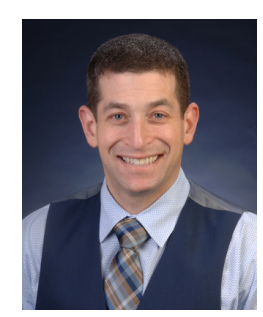

Dr. Brian A. Weiss has a B.S. in Mechanical Engineering (2000), Masters in Engineering (2003), and Ph.D. in Mechanical Engineering (2012) from the University of Maryland, College Park, Maryland. He is currently the Project Leader of the Prognostics, Health Management, and Control (PHMC) project within the Engineering Laboratory (EL) at the National Institute of Standards and Technology (NIST). Prior to his leadership role in the PHMC project, he spent 15 years conducting performance assessments across numerous military and first response technologies including autonomous unmanned ground vehicles; tactical applications operating on Android devices; advanced soldier sensor technologies; free-form, two-way, speech-to-speech translation devices for tactical use; urban search and rescue robots; and bomb disposal robots. He is currently a member of the American Society of Mechanical Engineers (ASME). His efforts have earned him numerous awards including a Department of Commerce Gold Medal (2013), Silver Medal (2011), Bronze Medals (2004 \& 2008), and the Jacob Rabinow Applied Research Award (2006). 